\title{
Von Willebrand factor and ADAMTS13 activity as clinical severity markers in patients with COVID-19
}

\author{
A Marco ${ }^{1,2}$ (D) P Marco ${ }^{1,2,3}$ (i) \\ Accepted: 13 April 2021 / Published online: 17 April 2021 \\ ( ) The Author(s), under exclusive licence to Springer Science+Business Media, LLC, part of Springer Nature 2021
}

\begin{abstract}
The coronavirus disease 2019 (COVID-19) increases thrombotic risk. The mechanisms that lead to this prothrombotic state are not well established. The main aim was to evaluate the von Willebrand factor (VWF) antigen and plasma ADAMTS13 activity as endothelial injury markers in COVID-19. We present a prospective study in COVID-19 patients recruited in our institution. VWF antigen, ADAMTS13 activity, D-dimer, and fibrinogen were measured during the first week once COVID19 was diagnosed. Fifty COVID-19 inpatients [44\% in the intensive care unit (ICU)] and 102 COVID-19 outpatients were enrolled. Thirty age and gender matched non-COVID-19 ward inpatients and 30 non-COVID-19 healthy individuals were recruited. The COVID-19 inpatients had higher D-dimer, fibrinogen, and VWF antigen levels and a lower ADAMTS13 activity compared with the COVID-19 outpatients $(\mathrm{p}<0.05)$. ICU patients had higher D-dimer and VWF antigen levels compared with the ward patients and the lowest ADAMTS13 activity $(\mathrm{p}<0.05)$. An imbalance in VWF antigen/ADAMTS13 ratio was observed in COVID-19, reaching the highest in ICU patients. In contrast to other ward non-COVID-19 inpatients, a significative reduction in ADAMTS13 activity was observed in all COVID-19 patients. There is an increase in VWF antigen and an ADAMTS13 activity reduction in COVID-19 related to disease severity and could predict poor clinical outcomes. The ADAMTS13 activity reduction could be a marker associated with COVID-19 compared to other non-critical medical conditions.
\end{abstract}

Keywords COVID-19 · Von Willebrand factor · ADAMTS13 $\cdot$ Endothelial dysfunction · Hypercoagulability

\section{Highlights}

- An endothelial disfunction is already present in early stages of COVID-19

- The VWF antigen increase and the ADAMTS13 activity reduction is related to COVID-19 severity

- ADAMTS13 activity reduction could be a marker associated with COVID-19 compared to other non-critical medical conditions.

A Marco

marcoana@hotmail.com

1 Thrombosis and Hemostasis Department, Hematology Service, University General Hospital in Alicante, Alicante, Spain

2 Biomedical Research Institute in Alicante, Alicante, Spain

3 Clinical Medicine Department, Miguel Hernández University, Alicante, Spain
- Endothelial lesion markers research in COVID-19 could provide a new therapeutic approach.

\section{Introduction}

The novel coronavirus disease 2019 (COVID-19) is caused by severe acute respiratory syndrome coronavirus 2 (SARSCoV-2) [1]. SARS-CoV-2 is highly infectious and contagious, and the high mortality rates worldwide represent an unmet challenge currently [2].

The development of coagulopathy is associated with poor prognosis. A procoagulant status in patients with pneumonia has been reported $[3,4]$.

An increase in D-dimer level is one of the most common hematological abnormalities found in hospitalized patients. Although older patients with comorbidities are known to have higher D-dimer levels, this abnormality in the context of COVID-19 promotes mortality due to COVID-19-derived complications [2]. Tang et al. 
described elevated D-dimer levels in non-survivors vs. survivors in COVID-19 [5]. Similarly, Huang et al. observed a higher D-dimer in patients requiring critical care support compared with patients who did not need it [6]. As a consequence, patients with markedly increased D-dimer levels should be strictly followed-up and consider hospitalization despite not having severe symptoms.

Fibrinogen levels remain elevated in the context of acute infection and predict mortality [7]. Thachil et al., instead of the prothrombotic role, discussed a protective function for fibrinogen. As an acute-phase reagent, the fibrinogen level is increased in patients with COVID-19 to protect the host [8]. Activated partial thromboplastin (APTT) is prolonged in few patients, probably due to the presence of lupus anticoagulant, which can induce thrombotic events [9]. Other abnormalities include slightly prolonged prothrombin time and occasional mild thrombocytopenia $[5,10,11]$.

However, the mechanisms that lead to this hypercoagulable state are not clearly defined. The previously described hematological abnormalities contrast with those described in disseminated intravascular coagulopathy [12]. Global hemostatic assays are consistent with a state of hypercoagulability. In COVID-19 patients, the thromboelastography showed an increased maximum clot firmness and a shorter clot formation time $[13,14]$ while the thrombin generation test showed a normal thrombin generation capacity despite the administration of heparin [14].

COVID-19 represents a severe inflammatory disease, and proinflammatory markers are released. Endothelial injury associated with a cytokine storm contributes to the thromboinflammatory process [15]. The von Willebrand factor (VWF) is a large multimeric glycoprotein stored in endothelial cells and platelets and released in response to different stimuli, such as acute inflammatory processes [16]. ADAMTS13 is a metalloprotease expressed by hepatic and endothelial cells and is implicated in the cleavage of prothrombotic ultra-large VWF multimers, contributing to the maintenance of hemostatic balance [12]. In this setting, acquired non-immune low ADAMTS13 levels could explain this thrombotic tendency. These findings could be related to ADAMTS13 consumption because of excess release of VWF, finally leading to thrombosis development. These clinical and laboratory data are compatible with thrombotic microangiopathy [17-19]. Therefore, it is crucial to clearly define coagulopathy to offer an appropriate therapeutic approach. Moreover, the mechanisms that induce this coagulopathy are not well established.

The main objective of our study was to evaluate the role of VWF antigen and plasma ADAMTS13 activity as endothelial injury markers in patients with COVID-19. The secondary objective was to analyze whether ADAMTS13 activity could be a prognostic marker associated with
COVID-19 compared to other non-critical acute medical conditions.

\section{Material and methods}

\section{Study design}

We performed a prospective observational study involving patients with COVID-19 confirmed by polymerase chain reaction during April and May 2020 in our institution.

The exclusion criteria were age below 18 years, current pregnancy, cancer, history of venous thromboembolism, and individuals receiving anticoagulant or antiplatelet therapy at inclusion.

The patients were divided into two groups depending on whether hospitalization was needed. Inpatients were subclassified into ward patients and those requiring intensive care.

All hospitalized patients received standard thromboprophylaxis with enoxaparin at a dose of $40 \mathrm{mg}$ daily and adjusted in case of renal impairment (glomerular filtration $<20 \mathrm{mg} / \mathrm{mL}$ ), in agreement with the ISTH and CHEST guidelines and recommendations [20,21].

We included two age and gender matched control groups recruited in our institution: (1) 30 non-COVID-19 ward inpatients with acute medical conditions and (2) 30 nonCOVID-19 healthy individuals.

The study was conducted in accordance with the basic principles of the World Medical Association Declaration of Helsinki and complied with the standards described in the European Union Guidelines for good clinical practice. This study was approved by the local ethics committee. All the patients provided signed informed consent prior to inclusion in the study.

\section{Sample processing}

Blood samples were obtained by direct vein puncture and anticoagulated with $0.129 \mathrm{M}$ sodium citrate during the first week once COVID-19 was confirmed. Platelet-poor plasma was immediately obtained using double centrifugation at $2500 \times g$ for $15 \mathrm{~min}$ at $22{ }^{\circ} \mathrm{C}$. The aliquots were stored at $-80^{\circ} \mathrm{C}$ until processing. The samples were defrosted at $37{ }^{\circ} \mathrm{C}$ for $10 \mathrm{~min}$ prior to analysis.

We measured the APTT ratio, international normalized ratio (INR), fibrinogen, and D-dimer levels with an automated coagulation analyzer (STA evolution, Stago, Paris, France) following the protocols from the manufacturer. Plasma ADAMTS13 activity and VWF antigen were measured using chemiluminescence (AcuStar, International Laboratory, Bedford, MA, USA) following the protocols from the manufacturer. The platelet count was measured with an automated analyzer (Sysmex, Roche, Basel, Switzerland). 


\section{Statistical analysis}

The qualitative variables were described as absolute and relative frequencies, and the quantitative variables were described as the median and interquartile range (p25-p75). We used the Mann-Whitney U test or $\chi^{2}$-test to compare the

Table 1 Characteristics of COVID-19 patients at inclusion

\begin{tabular}{lccr}
\hline & Inpatients $(\mathrm{n}=50)$ & $\begin{array}{l}\text { Outpatients } \\
(\mathrm{n}=102)\end{array}$ & $\mathrm{p}$-value \\
\hline Age (years) & $68.39(61.43-79.05)$ & $60.95(48.30-69.03)$ & $<0.05$ \\
Male n (\%) & $34(68)$ & $60(59)$ & 0.12 \\
Hypertension & $31(62)$ & $36(35)$ & $<0.05$ \\
Diabetes mellitus & $16(32)$ & $17(16)$ & $<0.05$ \\
\hline
\end{tabular}

Quantitative data are shown as median (p25-p75)

Qualitative data are shown as number (percentage) differences between groups where appropriate. Statistical significance was set at $5 \%(\mathrm{p}<0.05)$. The statistical software used was IBM SPSS version 20 (Chicago, IL, USA).

\section{Results}

We included 152 COVID-19 patients. One-hundred and two patients were followed-up in the outpatient clinic and 50 patients required hospitalization, 22 of whom were admitted to the ICU.

The characteristics of patients at inclusion are shown in Table 1.

Hemostatic parameters are described in Table 2. We observed a significant higher D-dimer, fibrinogen, and VWF antigen levels and a significant lower ADAMTS13 activity in COVID-19 inpatients than in COVID-19 outpatients. The VWF antigen/ADAMTS13 activity ratio

Table 2 Hemostatic parameters including control groups and depending on whether patients required hospital admission

\begin{tabular}{|c|c|c|c|c|c|}
\hline Parameter & $\begin{array}{l}\text { Non-COVID-19 } \\
\text { inpatients }(n=30)\end{array}$ & $\begin{array}{l}\text { Non-COVID-19 } \\
\text { healthy individuals } \\
(\mathrm{n}=30)\end{array}$ & $\begin{array}{l}\text { COVID-19 inpatients } \\
(\mathrm{n}=50)\end{array}$ & $\begin{array}{l}\text { COVID-19 Outpatients } \\
(\mathrm{n}=102)\end{array}$ & $\mathrm{p}$-value \\
\hline D-dimer $(\mu \mathrm{g} / \mathrm{mL})$ & $2.3(0.4-1)$ & $0.61(0.33-0.94)$ & $2.48(0.88-6.86)$ & $0.4(0.27-0.56)$ & $\begin{array}{l}<0.05^{*} \\
<0.05^{\text {** }} \\
<0.05^{\text {**** }} \\
0.93^{\text {***** }} \\
<0.05^{\text {****** }}\end{array}$ \\
\hline Fibrinogen $(\mathrm{mg} / \mathrm{dL})$ & $468(323-381)$ & $312(210-250)$ & $511(395-568)$ & $346.5(291-374)$ & $\begin{array}{l}<0.05^{*} \\
<0.05^{\text {** }} \\
<0.05^{\text {**** }} \\
<0.05^{\text {**** }} \\
<0.05^{\text {****** }}\end{array}$ \\
\hline ADAMTS13 activity (\%) & $85.1(68.5-107.6)$ & $99.9(82.4-112.5)$ & $44.4(32.5-60.8)$ & $59.9(43.4-78.75)$ & $\begin{array}{l}<0.05^{*} \\
<0.05^{\text {** }} \\
<0.05^{\text {**** }} \\
<0.05^{\text {**** }} \\
<0.05^{\text {****** }}\end{array}$ \\
\hline VWF antigen (\%) & $211.2(132.2-311.2)$ & $145.3(103-176)$ & $337.8(270.0-394.9)$ & $121.6(95.75-151.95)$ & $\begin{array}{l}<0.05^{*} \\
<0.05^{* *} \\
<0.05^{* * *} \\
0.19^{* * * *} \\
<0.05^{\text {****** }}\end{array}$ \\
\hline $\begin{array}{l}\text { VWF antigen/ } \\
\text { ADAMTS13 activity } \\
\text { ratio }\end{array}$ & $2.58(1.46-3.99)$ & $1.41(1.20-1.95)$ & $7.67(4.67-13.68)$ & $2.11(1.39-3.17)$ & $\begin{array}{l}<0.05^{*} \\
<0.05^{* *} \\
0.25^{* * *} \\
<0.05^{\text {**** }} \\
<0.05^{\text {****** }}\end{array}$ \\
\hline
\end{tabular}

Data are expressed as median (p25-p75)

$V W F$ antigen von Willebrand factor antigen

*p value: between COVID-19 inpatients and COVID-19 outpatients

** p value: between COVID-19 inpatients and non-COVID-19 inpatients

*** p value: between COVID-19 outpatients and non-COVID-19 inpatients

${ }^{* * * *}$ p value: between COVID-19 outpatients and non-COVID-19 healthy individuals

***** p value: between COVID-19 inpatients and non-COVID-19 healthy individuals 
was significantly higher in COVID-19 inpatients than in COVID-19 outpatients.

A similar trend with statistical significance was detected between COVID-19 inpatients and non-COVID-19 inpatients. The VWF antigen/ADAMTS 13 activity ratio was significantly higher in COVID-19 inpatients than in nonCOVID-19 inpatients. However, between COVID-19 outpatients and non-COVID-19 inpatients, we observed a lower ADAMTS13 activity and a reduction in fibrinogen and VWF antigen levels in COVID-19 outpatients $(\mathrm{p}<0.05)$. D-dimer remained similar in both groups.

We also described the highest ADAMTS13 activity and the lowest VWF antigen/ADAMTS13 ratio and fibrinogen levels in non-COVID-19 healthy individuals than in COVID-19 patients (see Table 2).

APTT, INR and platelet count remained within the normal range in all groups.
In addition, we compared these parameters in COVID-19 inpatients depending on whether intensive care was required. We observed significantly higher D-dimer and VWF antigen levels and significantly lower ADAMTS13 activity in ICU patients than in ward patients (see Table 3).

Furthermore, we evaluated changes in endothelial injury markers (ADAMST13 activity and VWF antigen) in survivors $(n=143)$ and non-survivors $(n=9)$ COVID-19 patients. ADAMTS13 activity was lower in COVID-19 non-survivors [39\% (24.7-44.2\%)] compared to COVID-19 survivors [55.45\% (40.4-72.9\%)], $\mathrm{p}<0.05$. VWF antigen was higher in COVID-19 non-survivors [370.2\% (312-400\%)] compared to COVID-19 survivors [143.5\% (104.5-218.9\%)], $\mathrm{p}<0.05$ (see Fig. 1).

We also studied the correlation between hemostatic parameters in COVID-19 patients. Of note, ADAMTS13 was inversely correlated with D-dimer, fibrinogen, and VWF antigen, all of which were statistically significant. In
Table 3 Hemostatic parameters in ward patients vs. ICU patients
Fig. 1 Median of ADAMTS13 activity and VWF antigen in survivors and non-survivors COVID-19 patients

\begin{tabular}{lccr}
\hline Parameter & Non-ICU $(\mathrm{n}=28)$ & ICU $(\mathrm{n}=22)$ & p-value \\
\hline D-dimer $(\mu \mathrm{g} / \mathrm{mL})$ & $0.89(0.73-2.31)$ & $4.64(2.66-11.04)$ & $<0.05$ \\
Fibrinogen $(\mathrm{mg} / \mathrm{dL})$ & $511(383-561)$ & $505(400-576)$ & 0.94 \\
ADAMTS13 activity $(\%)$ & $46.5(40.4-60.9)$ & $38.85(26-60)$ & $<0.05$ \\
VWF antigen $(\%)$ & $279.95(217.15-345.15)$ & $368.6(336.3-400)$ & $<0.05$ \\
VWF antigen/ADAMTS13 activ- & $5.67(3.40-9.63)$ & $11.28(6.08-19.11)$ & $<0.05$ \\
$\quad$ & & & \\
ity ratio & & &
\end{tabular}

Data are expressed as mean and a confidence interval of $95 \%$

$V W F$ antigen von Willebrand factor antigen

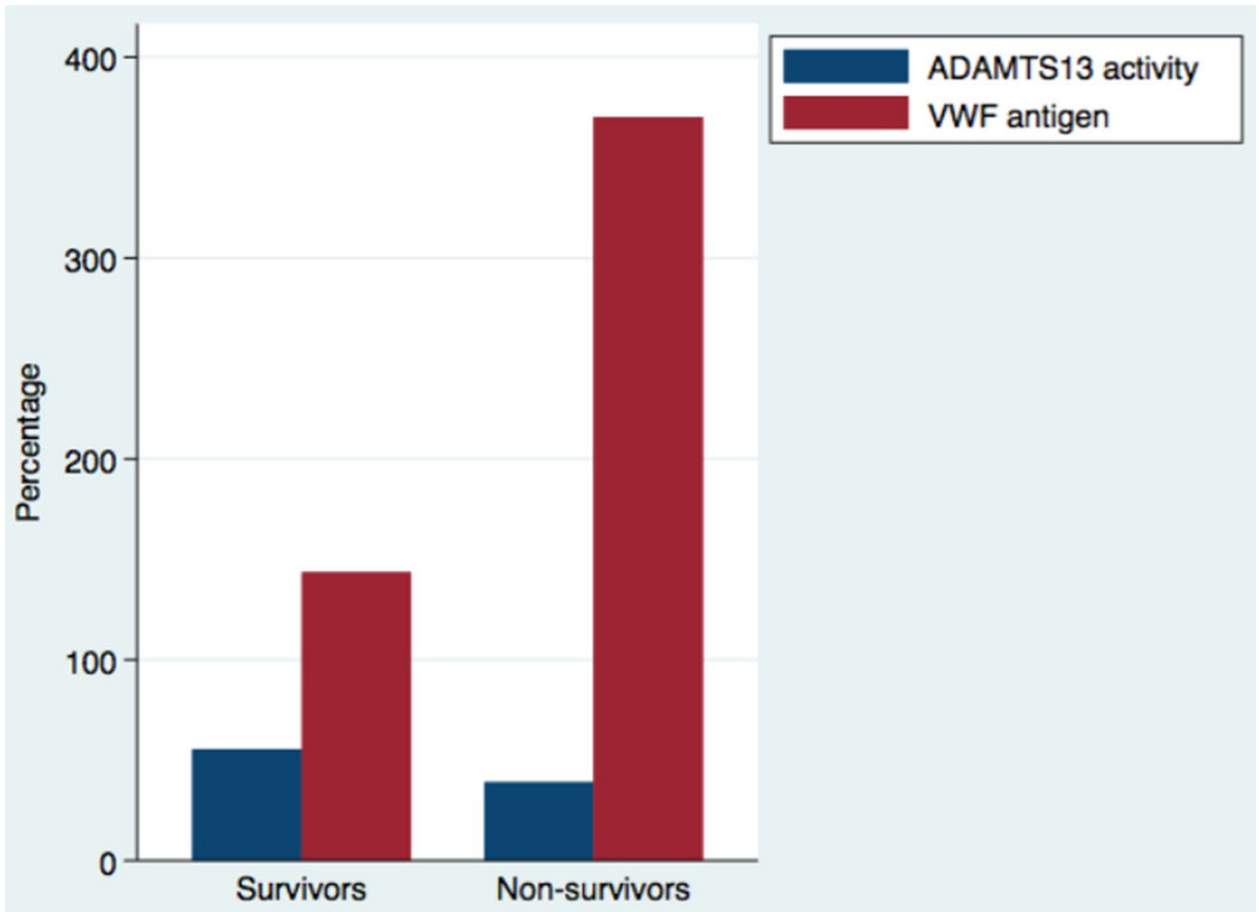


addition, VWF antigen presented a direct significant correlation, with both D-dimer and fibrinogen. Platelets also had a significant direct correlation with ADAMTS13 and an inverse correlation with VWF antigen (see Table 4).

ADAMTS13 activity was inversely correlated with hypertension, diabetes mellitus and age, while VWF antigen was directly correlated with these cardiovascular risk factors (see Table 5).

\section{Discussion}

COVID-19-associated coagulopathy has been broadly discussed in the literature. This coagulopathy overlaps with disseminated intravascular coagulopathy (DIC) in critically ill patients with circulatory collapse and multi-organ failure. However, they usually do not meet the ISTH criteria for DIC $[2,22]$.

There is an association between inflammation and coagulation. A noteworthy cytokine release, mainly IL-6, induces tissue factor overexposure in endothelial cells and monocytes, triggering thrombin generation. This cytokine storm correlates with poor outcome [23, 24].

In patients with COVID-19, as confirmed in our study, there is a peculiar coagulopathy derived from the systemic inflammatory response associated with endothelial dysfunction [12].

We found a higher D-dimer level in COVID-19 inpatients than in COVID-19 outpatients. This was significantly higher in patients requiring intensive care than in ward patients. Consequently, D-dimer predicts poor outcome as the highest levels are present in critically ill patients $[5,6]$. We would also like to remark that D-dimer was significantly increased in COVID-19 inpatients than in non-COVID-19 inpatients. Although D-dimer increases in hospitalized patients, it could be a hemostatic parameter of clinical worsening in COVID19 , implying hospitalization.

VWF and ADAMTS13 are expressed by endothelial cells. Dysregulation of the vascular endothelium in acute hyperinflammation setting induces excess VWF released by endothelial cells. ADAMTS13 activity decreases in proportion to the inflammatory response [12]. According to Katneni et al., this contrast between VWF and ADAMTS13 activity could be a consequence of inhibition and/or deficiency of ADAMTS13 activity [12]. In our study, ICU patients presented the lowest ADAMTS13 activity and the most elevated VWF antigen levels, with statistical significance between ward patients and ICU patients. Similarly, we found a significantly lower ADAMTS13 activity and more elevated VWF antigen in COVID-19 inpatients than in COVID-19 outpatients. We also described a lower ADAMTS13 activity and a higher VWF antigen in COVID-19 inpatients compared with non-COVID-19 inpatients. However, when studying COVID-19 outpatients and non-COVID-19 inpatients, we detected a statistical reduction in ADAMTS13 activity in COVID-19 outpatients. VWF antigen and fibrinogen levels were reduced in COVID-19 outpatients compared with nonCOVID-19 inpatients, probably because these parameters
Table 4 Correlation and statistical significance between hemostatic parameters in COVID-19 patients
Table 5 Correlation and statistical significance between endothelial injury markers and cardiovascular risk factors in COVID-19 patients

\begin{tabular}{lllll}
\hline & D-dimer $(\mu \mathrm{g} / \mathrm{mL})$ & Fibrinogen $(\mathrm{mg} / \mathrm{dL})$ & $\begin{array}{l}\text { ADAMTS13 } \\
\text { activity }(\%)\end{array}$ & VWF antigen (\%) \\
\hline Fibrinogen $(\mathrm{mg} / \mathrm{dL})$ & $\mathrm{r}=0.14$ & & & \\
& $\mathrm{p}<0.05$ & & & \\
ADAMTS13 activity $(\%)$ & $\mathrm{r}=-0.19$ & $\mathrm{r}=-0.28$ & & \\
& $\mathrm{p}<0.05$ & $\mathrm{p}<0.05$ & $\mathrm{r}=-0.32$ & \\
VWF antigen (\%) & $\mathrm{r}=0.55$ & $\mathrm{r}=0.54$ & $\mathrm{p}<0.05$ & \\
& $\mathrm{p}<0.05$ & $\mathrm{p}<0.05$ & $\mathrm{r}=0.20$ & $\mathrm{r}=-0.25$ \\
Platelets & $\mathrm{r}=-0.01$ & $\mathrm{r}=0.01$ & $\mathrm{p}<0.05$ & $\mathrm{p}<0.05$ \\
\hline
\end{tabular}

\begin{tabular}{lllll}
\hline & $\begin{array}{l}\text { ADAMTS13 } \\
\text { activity (\%) }\end{array}$ & VWF antigen (\%) & Hypertension & Diabetes mellitus \\
\hline VWF antigen (\%) & $\mathrm{r}=-0.32$ & & & \\
Hypertension & $\mathrm{p}<0.05$ & & & \\
Diabetes mesllitus & $\mathrm{r}=-0.23$ & $\mathrm{r}=0.26$ & & \\
& $\mathrm{p}=0.11$ & $\mathrm{p}<0.05$ & $\mathrm{r}=0.40$ & \\
Age & $\mathrm{r}=-0.21$ & $\mathrm{r}=0.23$ & $\mathrm{p}<0.05$ & $\mathrm{r}=0.20$ \\
& $\mathrm{p}=0.21$ & $\mathrm{p}=0.12$ & $\mathrm{r}=0.26$ & $\mathrm{p}=0.11$ \\
\hline
\end{tabular}


are acute reactive markers which are normally increased in hospitalized patients.

In our COVID-19 patients, the combination of low ADAMTS13 activity and elevated VWF levels may contribute to a microangiopathic state, more accentuated in critically ill patients and non-survivors. In agreement with our results, we consider that the decrease in ADAMTS13 activity could be due to hyper consumption of this protein when trying to break high molecular weight VWF multimers.

To emphasize this endothelial injury, the VWF antigen/ ADAMTS 13 activity ratio was especially higher in patients requiring intensive care support than in ward patients (11.28 vs. 5.67) and similarly in COVID-19 inpatients vs. COVID19 outpatients (7.67 vs. 2.11). Although this ratio was also increased in non-COVID-19 inpatients, it may be due to higher VWF levels in the setting of other acute inflammatory processes. Overall, there is a disproportion between VWF antigen and ADAMTS13 activity that could predict a poor prognosis in COVID-19 patients. This means that the higher the VWF antigen/ADAMTS13 activity ratio, the poorer the clinical outcome.

We would like to remark that the median ADAMTS13 activity in COVID-19 outpatients was $59.9 \%$, decreased to $46 \%$ in ward patients, and reached the lowest levels in ICU patients (38\%), with statistical significance between groups. This implies a significative ADAMTS13 decrease in COVID-19 patients and contributes to explaining the procoagulant tendency. This reduction is accentuated according to clinical severity. On the contrary, ADAMTS13 activity remained within normality in non-COVID-19 ward inpatients and in healthy individuals.

To reinforce this procoagulant condition, we studied the correlation between different hemostatic parameters in COVID-19 patients. ADAMTS13 was inversely correlated with D-dimer, fibrinogen, and VWF antigen, all of which were statistically significant. In addition, VWF antigen presented a significant direct correlation with both D-dimer and fibrinogen. Of note, the strongest correlations were found between VWF antigen and D-dimer and between fibrinogen and VWF antigen, which are normally increased in acute inflammatory processes. In addition, the inverse correlation between ADAMTS13 and VWF antigen enhances the microangiopathy state. Moreover, although no statistical differences between groups were found when evaluating platelet count, we found a direct correlation between ADAMTS13 activity and platelets and an inverse correlation between VWF antigen levels and platelets.

Mancini et al. has recently published that an alteration in the VWF-ADAMTS13 axis in COVID-19 patients is related to disease severity. They designed a cross-sectional study including 50 critical admitted to 3 units of different intensity care and recruited 274 healthy volunteers tested for thrombophilia as control group [25]. However, we performed a prospective study and patients were divided into two groups depending on whether hospitalization was needed. Inpatients were subclassified into ward patients and those requiring intensive care. In agreement with Mancini et al., we found an imbalance in the VWF/ADAMTS13 ratio, more accentuated in hospitalized patients, mainly those in the ICU. On top of that, in our study, patients that did not require hospitalization also had a discrepancy in the VWF/ADAMTS13 ratio (ratio of 2.11), and this ratio increased in ward patients and reached the highest levels in ICU patients. That means that all COVID-19 patients, even those at early stages of the disease, referred a disproportion between VWF and ADAMTS13 activity. This VWF/ADAMTS13 ratio could be considered an evolutionary prognostic marker of clinical deterioration. In addition, we described an inverse correlation between VWF antigen and ADAMTS13 that could reinforce the microangiopathic state.

Our COVID-19 inpatients had a higher incidence of hypertension and diabetes and were older than COVID-19 outpatients. The combination of procoagulant parameters and the presence of cardiovascular risk factors promotes vascular inflammation and endothelial dysfunction. In fact, we found that these clinical factors inversely correlated with ADAMTS13 activity and directly correlated with VWF antigen.

The main strength of our study is that it is based on a broad prospective cohort of patients with COVID-19 in a single reference center for 2 months. In addition, we avoided possible confounding factors by excluding patients with hypercoagulable states (see exclusion criteria) and those treated with anticoagulants or antiplatelet treatments at inclusion. We also recruited 2 control groups, both inpatients and healthy individuals without COVID-19.

Our study includes the largest series in the literature that evaluates both VWF and ADAMTS13 in COVID-19 patients with different degrees of clinical severity. However, a multi-centric study with greater recruitment could reinforce our findings.

To conclude, the imbalance between VWF antigen and ADAMTS 13 activity, expressed as a ratio between the two proteins is related to the severity of COVID-19 and could be potential prediction markers for poor clinical outcome. This imbalance was also observed in COVID-19 patients that did not require hospitalization.

In addition, ADAMTS13 activity could be a potential prognostic marker associated with COVID-19 compared to other non-critical acute medical diseases.

Author contributions AM wrote the paper, designed the database, and performed the statistical analysis. PM designed and supervised the study. All authors read and approved the final version of the manuscript. 


\section{Declarations}

Conflict of interest The authors declare there are no conflicts of interest.

\section{References}

1. Wu D, Wu T, Liu Q, Yang Z (2020) The SARS-CoV-2 outbreak: what we know. Int J Infect Dis 94:44-48

2. Thachil J, Tang N, Gando S et al (2020) ISTH interim guidance on recognition and management of coagulopathy in COVID-19. J Thromb Haemost 18:1023-1026

3. Connors JM, Levy JH (2020) Thromboinflammation and the hypercoagulability of COVID-19. J Thromb Haemost 18:1559-1561

4. Ranucci M, Ballotta A, Di Dedda U et al (2020) The procoagulant pattern of patients with COVID-19 acute respiratory distress syndrome. J Thromb Haemost 18:1747-1751

5. Tang N, Li D, Wang X, Sun Z (2020) Abnormal coagulation parameters are associated with poor prognosis in patients with novel coronavirus pneumonia. J Thromb Haemost 18:844-847

6. Huang C, Wang Y, Li X et al (2020) Clinical features of patients infected with 2019 novel coronavirus in Wuhan China. Lancet 395:497-506

7. Zhang Y, Cao W, Jiang W et al (2020) Profile of natural anticoagulant, coagulant factor and anti-phospholipid antibody in critically ill COVID-19 patients. J Thromb Thrombolysis 50:580-586

8. Forum TJ (2020) The protective rather than prothrombotic fibrinogen in COVID-19 and other inflammatory states. J Thromb Haemost 18:1849-1852

9. Bowles L, Platton S, Yartey N et al (2020) Lupus anticoagulant and abnormal coagulation tests in patients with COVID-19. N Engl J Med 383:288-290

10. Lippi G, Plebani M, Henry BM (2020) Thrombocytopenia is associated with severe coronavirus disease 2019 (COVID-19) infections: a meta-analysis. Clin Chim Acta 506:145-148

11. Panigada M, Bottino N, Tagliabue P et al (2020) Hypercoagulability of COVID-19 patients in the intensive care unit: a report of the thromboelastography findings and other parameters of hemostasis. J Thromb Haemost 18:1738-1742

12. Katneni UK, Alexaki A, Hunt RC et al (2020) Coagulopathy and thrombosis as a result of severe COVID-19 infection: a microvascular focus. Thromb Haemost. https://doi.org/10.1055/s-00401715841

13. Pavoni V, Gianesello L, Pazzi M, Stera C, Meconi T, Frigieri FC (2020) Evaluation of coagulation function by rotation thrombelastometry in critically ill patients with severe COVID19 pneumonia. J Thromb Thrombolysis 50:281-286
14. Blasi A, Von Meijenfeldt FA, Adelmeijer J et al (2020) In vitro hypercoagulability and ongoing in vivo activation of coagulation and fibrinolysis on COVID-19 patients on anticoagulation. $\mathrm{J}$ Thromb Haemost 18:2646-2653

15. White D, MacDonald S, Edwards T et al (2020) Evaluation of COVID-19 coagulopathy; laboratory characterization using thrombin generation and nonconventional haemostasis assays. Int J Lab Hematol. https://doi.org/10.1111/IJLH.13329

16. Gragnano F, Sperlongano S, Golia E et al (2017) The role of von Willebrand factor in vascular inflammation: from pathogenesis to targeted therapy. Mediators Inflamm 2017:5620314

17. Bazzan M, Montaruli B, Sciascia S, Cosseddu D, Norbiato C, Roccatello D (2020) Low ADAMTS13 plasma levels are predictors of mortality in COVID-19 patients. Intern Emerg Med $15: 861-863$

18. Huisman A, Beun R, Sikma M, Westerink J, Kusadasi N (2020) Involvement of ADAMTS13 and von Willebrand factor in thromboembolic events in patients infected with SARS-CoV-2. Int J Lab Hematol 42:211-212

19. Adam EH, Zacharowski K, Miesbach W (2020) A comprehensive assessment of the coagulation profile in critically ill COVID-19 patients. Thromb Res 194:42-44

20. Spyropoulos AC, Levy JH, Ageno W et al (2020) Scientific and standardization committee communication: clinical guidance on the diagnosis, prevention, and treatment of venous thromboembolism in hospitalized patients with COVID-19. J Thromb Haemost 18:1859-1865

21. Moores LK, Tritschler T, Brosnahan S et al (2020) Prevention, diagnosis, and treatment of venous thromboembolism in patients with COVID-19: CHEST guideline and expert panel report. Chest 158:1143-1163

22. Wada H, Thachil J, Di Nisio M et al (2013) Guidance for diagnosis and treatment of DIC from harmonization of the recommendations from three guidelines. J Thromb Haemost 11:761-767

23. Hu B, Huang S, Yin L (2020) The cytokine storm and COVID-19. J Med Virol. https://doi.org/10.1002/jmv.26232

24. Ye Q, Wang B, Mao J (2020) The pathogenesis and treatment of the cytokine storm in COVID19. J Infectol 80:607-613

25. Mancini I, Baronciani L, Artoni A et al (2020) The ADAMTS13von Willebrand factor axis in COVID-19 patients. J Thromb Haemost. https://doi.org/10.1111/JTH.15191

Publisher's Note Springer Nature remains neutral with regard to jurisdictional claims in published maps and institutional affiliations. 